Research Paper

\title{
Differential microRNA Profiles Predict Diabetic Nephropathy Progression in Taiwan
}

\author{
Hung-Yu Chien ${ }^{1 *}$, Chang-Yi Chen ${ }^{2 *}$, Yen-Hui Chiu ${ }^{3}$, Yi-Chun Lin ${ }^{4,5}$, Wan-Chun Li²,3凶 \\ 1. Department of Endocrinology \& Metabolism, Taipei City Hospital, Ren-Ai Branch, Taipei, Taiwan; \\ 2. Institute of Oral Biology and Department of Dentistry, School of Dentistry, National Yang-Ming University, Taipei, Taiwan; \\ 3. Department of Education and Research, Taipei City Hospital, Taipei, Taiwan; \\ 4. Division of Endocrinology \&Metabolism, Taipei Veterans General Hospital, Taipei, Taiwan: \\ 5. Faculty of Medicine, National Yang-Ming University, Taipei, Taiwan; \\ *These authors contributed equally.
}

$\square$ Corresponding author: Wan-Chun Li, Ph.D. Institute of Oral Biology and Department of Dentistry, School of Dentistry, National Yang-Ming University, No.155, Sec.2, Li-Nong St, Taipei, 11221, Taiwan. Phone: +886-2-28267255; Fax: +886-2-28264053; E-mail: wcli@ym.edu.tw.

() Ivyspring International Publisher. Reproduction is permitted for personal, noncommercial use, provided that the article is in whole, unmodified, and properly cited. See http://ivyspring.com/terms for terms and conditions.

Received: 2016.03.15; Accepted: 2016.05.09; Published: 2016.06.01

\begin{abstract}
Objectives: Diabetic nephropathy (DN) is a major leading cause of kidney failure. Recent studies showed that serological microRNAs (miRs) could be utilized as biomarkers to identify disease pathogenesis; the DN-related miRs, however, remained to be explored. Methods: A prospective case-control study was conducted. The clinical significance of five potential miRs (miR-21, miR-29a, miR-29b, miR-29c and miR 192) in type 2 Diabetes Mellitus (T2DM) patients who have existing diabetic retinopathy with differential Albumin:Creatinine Ratio (ACR) and estimated Glomerular Filtration Rate (eGFR) was performed using quantitative RT-PCR analysis. The subjects with diabetic retinopathy enrolled in Taipei City Hospital, Taiwan, were classified into groups of normal albuminuria $(A C R<30 \mathrm{mg} / \mathrm{g} ; \mathrm{N}=12)$; microalbuminuria $(30 \mathrm{mg} / \mathrm{g}<A C R<300 \mathrm{mg} / \mathrm{g} ; \mathrm{N}=17)$ and overt proteinuria $(A C R>300 \mathrm{mg} / \mathrm{g} ; \mathrm{N}=21$ ) as well as $18 \mathrm{low}$-eGFR (eGFR $<60 \mathrm{ml} / \mathrm{min})$ and 32 high-eGFR (eGFR $>60 \mathrm{ml} / \mathrm{min}$ ). The level of serum miRs was statistically correlated with age, Glucose AC, ACR, eGFR and DN progression. Results: The levels of miR-21, miR-29a and miR-192 were significantly enriched in the overt proteinuria group compared with microalbuminuria and/or overt proteinuria groups. It was shown that only miR-21 level was significantly up-regulated in low-eGFR group compared with high-eGFR patients. Interestingly, Pearson's correlation coefficient analysis demonstrated that DN progressors showed significantly greater levels of miR-21, miR-29a, miR-29b and miR-29c in comparison with non-progressors implying the clinical potential of DN associated miRs in monitoring and preventing disease advancement. Conclusion: Our findings showed that miR-21, miR-29a/b/c and miR-192 could reflect DN pathogenesis and serve as biomarkers during $\mathrm{DN}$ progression.
\end{abstract}

Key words: Albumin:creatinine ratio, Biomarkers, Circulating microRNA, Diabetic nephropathy, Estimated Glomerular Filtration Rate.

\section{Introduction}

Over past decades, the incidence of type 2 diabetes mellitus (T2DM) has increased exponentially, especially in developed countries [1, 2]. Many studies have found that DM mediated microvascular and macrovascular pathological conditions could result in different complications leading to a great morbidity in T2DM subjects [3, 4]. It is estimated approximate $40 \%$ of T2DM patients develop diabetic nephropathy (DN) and the current clinical managements of $\mathrm{DN}$ are imperfect in delaying disease process and around $40 \%$ of DN subjects progress to end-stage renal disease [5]. The pathogenesis of DN involves the deregulation of various biological functions such as endothelial dysfunction, oxidative stress and the excess accumulation and deposition of extracellular matrix (ECM) in kidney [6]. 
The clinical features of DN include persistent albuminuria and progressive decline of glomerular filtration rates (GFR). While microalbuminuria (30-300 mg/day) could be detected in early, reversible DN subjects, overt proteinuria (>300 mg/day) represents an irreversible stage of $\mathrm{DN}[7,8]$. The detection of microalbuminuria is the standard method for the diagnosis of early-staged DN; however, it has some drawbacks. For example, microalbuminuria can develop when advanced pathological conditions have already been established, as assessed by renal biopsy examinations. In addition, it was found that the degree of albuminuria does not closely correlate with the GFR decrement suggesting the unreliability to utilize urinary albumin content as DN prognostic indicator [9]. It therefore becomes essential to define the early molecular changes in DM patients who are prone to develop progressive renal complications, defined as progressor, compared with non-progressor in order to monitor disease status in-time. Indeed, previous studies using proteomic analysis have defined candidate diagnostic proteins in urine from normoalbuminuric T2DM subjects who subsequently developed DN. However, the procedure is less than ideal to monitor the DN progression because it is time-consuming and could only target proteins abundantly expressed in urine $[10,11]$. Thus, more sensitive biomarkers with relative minimal invasive sampling and simple experimentation procedure for detection of early staged DN are particularly desirable.

In addition to serological or urinary proteins, recent studies have found that circulating nucleic acids represented potential indicators for disease diagnosis $[12,13]$. Among them, microRNA $(\mathrm{miR})$ is a new class of small single-stranded RNAs. Over 3,500 miRs have been discovered in the human genome and more than $90 \%$ of protein encoding mRNAs could be regulated by miRs in a tissue- and/or cell-specific manner [14]. miRs are 19-25-nucleotide-long non-coding RNA molecules that normally bind to the $3^{\prime}$ untranslated region ( $3^{\prime}$-UTR) of their target mRNAs leading to mRNA degradation and/or translational inhibition [15-17]. As a result, miRs could regulate a wide range of biological events and onset/progression of different diseases [18]. Although miR in serum or plasma seems promising to be utilized as a biomarker to identify disease initiation and progression, compared with cancer and cardiovascular disease, far less is known about the relevance of circulating miRs in DM complications. The significance of circulating miRs to delineate pathogenesis of DN was still poorly understood even though the potential DN-related miRs are elucidated in recent studies. Some controversial data and lack of information on human subjects, however, limited their usefulness as biomarker for diagnosis of DN progression.

In the present study, we therefore conducted a case control study to examine differential levels of a number of candidate circulating miRs from different staged DN patients, with coexisting diabetic retinopathy, aiming to identify the early predictor for DN progression. Recent studies have defined the importance of miR-21, miR-29 family and miR-192 in normal renal development, kidney function and the association of various renal diseases suggesting that these miRs might be good targets to monitor DN pathology. To the best of our knowledge, to date, no studies were yet reported to define the correlation between the level of these potential DN-related miRs and the disease progression further highlighting the requirement to examine the association of the serological miRs and DN pathogenesis in human subjects.

\section{Materials and Methods}

\section{Study population}

Patient sera were collected under the approval of Institutional Review Board (IRB) of Taipei City Hospital, Taiwan (TCHIRB-1010718). All patients were recruited from the Endocrinology and Metabolism outpatient clinic and divided into three groups according to albumin:creatinine ratio (ACR, $\mathrm{mg} / \mathrm{g}$ ) or estimated glomerular filtration rate (eGFR; $\mathrm{ml} / \mathrm{min}$ ) and followed up longitudinally at least for 2 years. The patients with ACR $<30 \mathrm{mg} / \mathrm{g}$ was classified as normoalbuminuria, the values of $30<A C R<300$ was defined as microalbuminuria while the group with ACR over $300 \mathrm{mg} / \mathrm{g}$ are overt proteinuria. To set a more stringent classification, the participants were divided into two groups: low-eGFR $(<60 \mathrm{ml} / \mathrm{min})$ and high-eGFR $(>60 \mathrm{ml} / \mathrm{min})$ based on values of eGFR. The MDRD method was used to estimate eGFR levels using a formula: $186 \times$ (serum creatinine level) $)^{-1.154} \mathrm{x}$ (age) ${ }^{-0.203} \times 0.742$ (if female). Patients with underlying malignancies, kidney disease history and other systemic diseases were excluded from this study. Renal sonographic analysis have been carried out by nephrologists to further verify DN. All of the patients had received fundoscopic examination to access for diabetic retinopathy and all $(21 / 21)$ of the patients in overt proteinuria group, all (17/17) of microalbuminuria group and 11/12 of normoalbuminuria group had coexisting diabetic retinopathy. All patients with DN received angiotensin converting enzyme inhibitors or angiotensin receptor blockers accordingly unless contraindicated. Other clinical criteria including age, 
glucose $\mathrm{AC}, \mathrm{HbAlc}$ and creatinine levels were also recorded to provide better evaluation for this study.

\section{Serum collection and microRNA isolation}

Serum was isolated from patients' peripheral blood and stored in RNase/DNase-free tubes at $-80^{\circ} \mathrm{C}$ until RNA isolation. Total RNA containing small RNA was isolated from $500 \mu \mathrm{l}$ of serum using mirVana isolation kit (Ambion) following the manufacturer's instructions with modifications. In brief, the collected serum was firstly mixed with Denaturing Solution containing 2-mercaptoethanol followed by the addition of Acid-Phenol/Chloroform and ethanol to precipitate total RNA and eluted in nuclease-free water. For small RNA isolation, total RNA was then incubated with miRNA Wash Solutions without pre-amplification and then eluted in appropriate volume of nuclease-free water. The samples were stored in $-20^{\circ} \mathrm{C}$ until use.

\section{Quantitative Real-Time Polymerase Chain Reaction (qRT-PCR) Analysis}

The amount of $10 \mu \mathrm{g}$ of $\mathrm{miR}$ was reverse transcribed to complementary DNA (cDNA) using the TaqMan MicroRNA Reverse Transcription Kit (Thermo Scientific) and miRNA-specific stem-loop primers following the manufacturer's instructions. The qPCR analysis was performed in duplicate with the ABI 7500 Fast Real-Time PCR system using the TaqMan Universal Master Mix II, no UNG kit (Thermo Fisher Scientific). All PCR primers were obtained from Thermo Scientific (Supplementary Table S1). Each reaction included cDNA template, TaqMan Universal Master Mix II, TaqMan Gene Expression Assay and nuclease-free water in the final volume of $20 \mu 1$. The internal control gene, non-coding small RNA U6 snRNA, was used according to the Applied Biosystems Application Note. The difference of $\mathrm{Ct}$ between the target miRs and U6 snRNA $(\Delta \mathrm{Ct})$ equivalent to the ratio of log2-transformed absolute copy numbers was employed to show the relative expression levels of the target miRNAs. The recommended reaction conditions were set according to the manufacturer's protocol $\left(50^{\circ} \mathrm{C}\right.$ for 2 mins, $95^{\circ} \mathrm{C}$ for 10 mins, 40 cycles of $95^{\circ} \mathrm{C}$ for 15 secs and $60^{\circ} \mathrm{C}$ for 1 mins).

\section{Statistical Analysis}

The unpaired t-test analysis was employed to compare age, glucose $\mathrm{AC}, \mathrm{HbA1C}$, Creatinine levels, ACR and eGFR among different subject groups. The statistical $\mathrm{P}$ value was generated by the one-way ANOVA analysis following Fisher's LSD post hoc multiple comparisons. Pearson correlation coefficient comparisons were carried out to determine disease progression rates and miR levels. Statistical evaluation was otherwise by $t$ test, using Microsoft Excel, with P_0.05 assumed to be significant. Statistical analysis was performed using statistical software program package Prism 5 (GraphPad, San Diego, CA). The statistical significance was defined as $\mathrm{P}<0.05$.

\section{Results}

\section{Candidate miRs were stably detected in serum from subject groups}

Fifty T2DM participants were enrolled in the study to determine DN-related miRs. Among them, 12 T2DM patients were normoalbuminuria, 17 of them are microalbuminuria and 21 subjects were diagnosed with overt proteinuria based on ACR classification whereas 32 of them exhibited high eGFR and 18 of them showed low eGFR levels. No significant difference in sex distribution, $\mathrm{HbA} 1 \mathrm{C}$ and/or age was found among subject groups with differential ACR (Table 1) and eGFR levels (Table 2). The choice of anticoagulant tubes was firstly verified to determine the potential interference of additives during sampling procedure. The results showed that the miR level was undetected in blood samples collected in sodium heparin containing tubes compared with the tubes without anticoagulant and sodium EDTA suggesting an inhibitory effect of anticoagulant for either RT or PCR experimental process (Supplementary Figure 1). Based on these data, we therefore collected whole blood in tube without anticoagulant and purified serum for further analysis. The validation using real-time RT-PCR analysis for miR level was also performed and none of the assays failed to reach the set threshold $\mathrm{Ct}$ of 40 cycles suggesting a reliable $\mathrm{Ct}$ values during analysis (Supplementary Figure 2).

Table 1. Clinical information of subjects recruited in this study categorized by Albumin Creatinine Ratio (ACR). *** $p<0.001$; $*_{p}<0.05$

\begin{tabular}{lllll}
\hline & $\begin{array}{l}\text { Normal } \\
\text { albuminuria }\end{array}$ & Microalbuminuria $\begin{array}{l}\text { Overt } \\
\text { proteinuria }\end{array}$ & P-Value \\
\hline Male & 9 & 12 & 13 & $>0.05$ \\
Female & 3 & 5 & 8 & $>0.05$ \\
Age (yrs) & $52.83 \pm 3.346$ & $54.35 \pm 2.602$ & $60.81 \pm 1.988$ & $<0.05^{*}$ \\
$\begin{array}{l}\text { Glucose AC } \\
\text { (mg/dl) }\end{array}$ & $156.6 \pm 15.67$ & $174.3 \pm 16.25$ & $128.6 \pm 7.825$ & $<$ \\
HbA1C (\%) & $8.000 \pm 0.4950$ & $7.91 \pm 0.3858$ & $7.176 \pm 0.2500$ & $>0.05$ \\
$\begin{array}{l}\text { Creatinine } \\
\text { (mg/dl) }\end{array}$ & $0.9833 \pm 0.1014$ & $0.8471 \pm 0.0692$ & $1.890 \pm 0.2844$ & $<0.0001^{* * *}$ \\
ACR (mg/g) & $13.86 \pm 2.690$ & $139.9 \pm 21.95$ & $1856 \pm 352.1$ & $<0.0001^{* * *}$ \\
eGFR & $88.36 \pm 9.465$ & $103.3 \pm 8.542$ & $54.24 \pm 8.378$ & $<0.0001^{* * *}$ \\
(ml/min) & & & & \\
\hline
\end{tabular}


Table 2. Clinical information of subjects recruited in this study divided by estimated Glomerular Filtration Rate (eGFR). $*_{* *} \mathrm{p}<0.001 ; *_{\mathrm{p}}<0.05$.

\begin{tabular}{llll}
\hline & High eGFR & Low eGFR & P-Value \\
\hline Male & 23 & 13 & \\
Female & 9 & 5 & \\
Age (yrs) & $55.44 \pm 1.79$ & $58.50 \pm 2.76$ & $>0.05$ \\
$\begin{array}{l}\text { Glucose AC } \\
\text { (mg/dl) }\end{array}$ & $165.3 \pm 10.43$ & $125.2 \pm 8.79$ & $<0.05^{*}$ \\
HbA1C (\%) & $7.90 \pm 0.27$ & $7.13 \pm 0.28$ & $>0.05$ \\
Creatinine & $0.83 \pm 0.04$ & $2.21 \pm 0.28$ & $<0.0001^{* * *}$ \\
(mg/dl) & & & \\
ACR (mg/g) & $510 \pm 181.5$ & $1404 \pm 397.6$ & $<0.05^{*}$ \\
eGFR (ml/min) & $103.4 \pm 5.41$ & $35.88 \pm 3.38$ & $<0.0001^{* * *}$ \\
\hline
\end{tabular}

\section{Levels of miR-21 and miR-29 are elevated in DN patients}

The level of miR-21, miR-29 family and miR-192 in serum samples from T2DM patients with differential ACR and eGFR levels were examined. The results showed that miR-21 level is more significantly
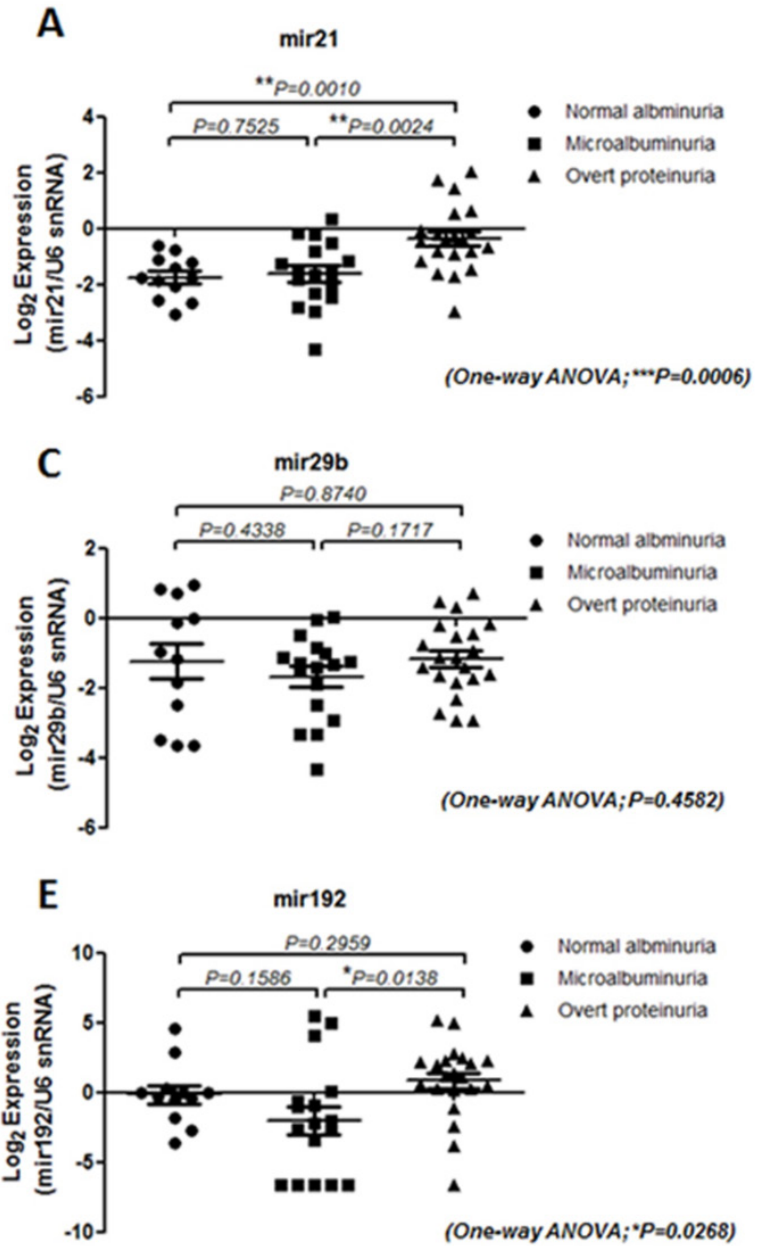

enriched in patients with overt proteinuria $(p=0.001)$ and microalbuminuria $(p=0.0024)$ than patients without DN whereas miR-29a level is up-regulated in subjects with more progressive DN compared with T2DM patients with normal albuminuria (Figure 1). Interestingly, although miR-192 level showed no significant difference between T2DM subjects with and without DN, the serological miR-192 levels differ between microalbuminuria and overt proteinuria groups $(p=0.0138)$ suggesting that miR-192 level might be a potential marker for late $\mathrm{DN}$ progression (Figure 1E). The miR level in patients with different eGFR was also analyzed. To our surprise, only miR-21 level is significantly up-regulated in subjects with lower eGFR (eGFR<60 ml/min; $p=0.0363)$ in comparison with patients with higher eGFR (eGFR $>60 \mathrm{ml} / \mathrm{min}$ ) whereas no difference were detected for level of miR-29 family and miR-192 between subject groups (Figure 2).
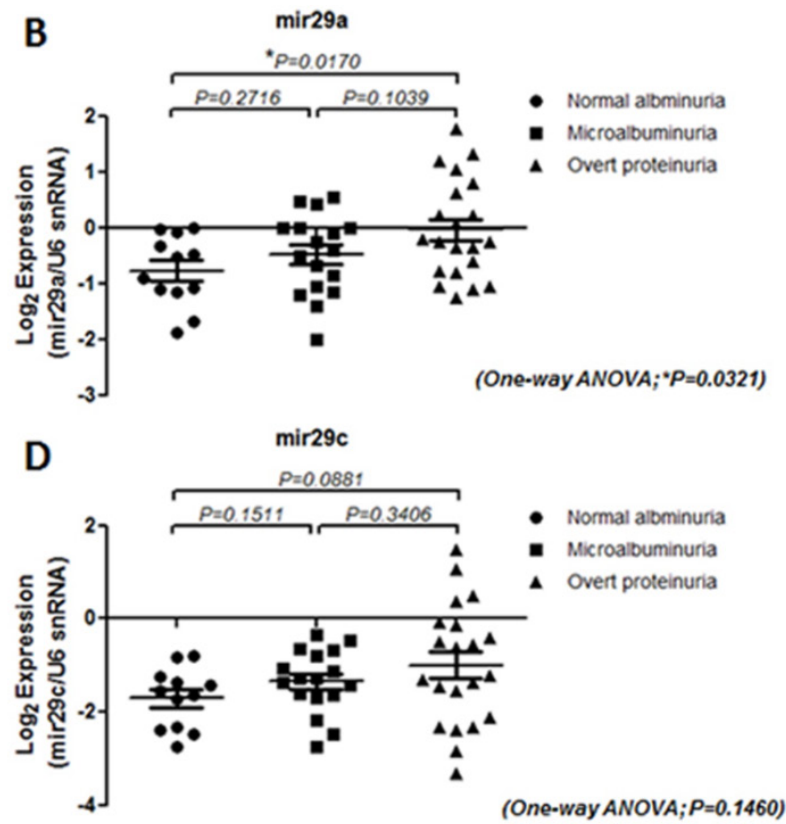

Figure 1. Expression of serum miRNAs in type 2 diabetic patients with differential ACRs. Quantitative real-time RT-PCR analysis of the five miRNAs in the sera from three groups (normal albuminuria, $n=12$; Microalbuminuria, $n=17$ and Overt proteinuria, $n=21$ ) showed that the level of miR-21, miR-29a and miR-192 is up-regulated in overt proteinuria subjects compared with normal or micro albuminuria groups. The $Y$-axis refers to the gene expression ratio (candidate miRs versus U6 snRNA), shown as the mean \pm SEM in log2 scale. Level was measured in triplicate and $P$ values were generated by one-way ANOVA analysis following Fisher's LSD post hoc multiple comparisons. **P $<0.01 ; * \mathrm{P}<0.05$. 
A

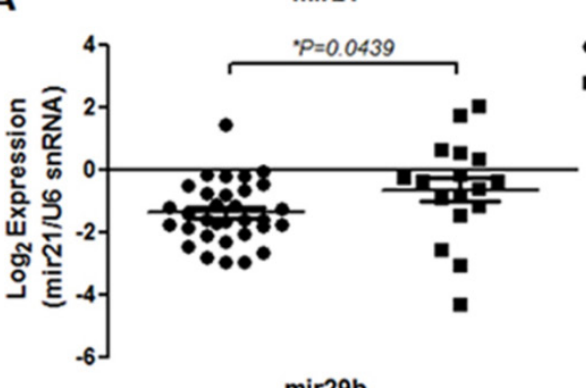

C

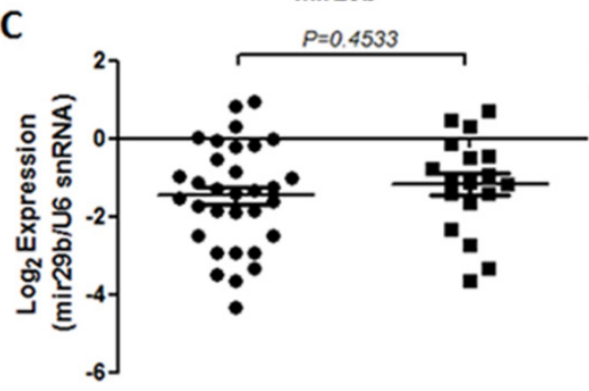

E

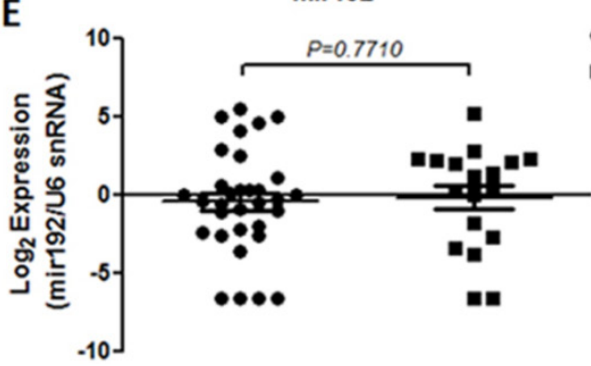

mir29a

B

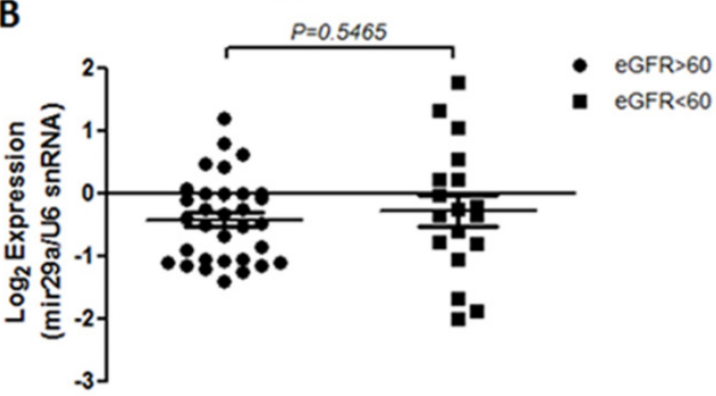

D

eGFR>60

- eGFr<60

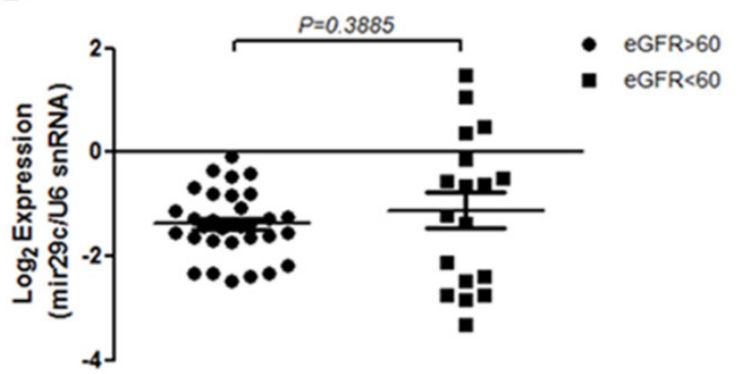

Figure 2. Level of serum miRNAs in type 2 diabetic patients with differential eGFR. Quantitative real-time RT-PCR analysis of the five miRs in the sera from two groups (high eGFR, $n=32$ and low eGFR, $n=18$ ) showed that only miR-21 level is up-regulated in low-eGFR groups compared with high-eGFR subjects. The $Y$-axis refers to the gene expression ratio (miRNAs versus U6 snRNA), shown as the mean \pm SEM in log2 scale. Expression was measured in triplicate, and $P$ values were generated by unpaired t-test analysis. $* \mathrm{P}<0.05$.

While the age and Glucose AC values differs among subject groups, the correlation between age and Glucose AC levels with the level of candidate miRs was next carried out. Pearson's correlation coefficient analysis showed that no significant associations were found between the level of target miRs with either age (Supplementary Figure 3) or Glucose AC values (Supplementary Figure 4) within all individuals implying that the differential circulating miR level is independent of patients' age and glycemic condition in this study.

\section{Level of miR-21 and miR-29 family reflected DN progression}

Based on our results showing that miR-21, miR-29 and miR-192 are potential biomarkers to detect staged $\mathrm{DN}$, it was found that a number of participants expressed an extremely high level of these target miRs. We suspected that these patients showed distinct pathological conditions different from the rest of subjects. To further evaluate this effect, the associations of disease progression defined by the annual change of plasma creatinine levels and level of miR-21, miR-29 family and miR-192 were analyzed using Pearson's correlation coefficient method. All participants were longitudinally followed up at least for 2 years and serial measures of serum creatinine were made every 2-3 months during the follow up period. Plasma creatinine changes were determined if two out of three consecutive creatinine measures were consistent and maintained. To our surprise, the results showed that the patients with greater levels of miR-21 and miR-29a/b/c, but not miR-192, displayed a more rapid elevation of creatinine levels in plasma suggesting that higher level of miR-21 and miR-29 family could possibly be prognostic markers for renal function impairment in T2DM patients (Figure 3). 
A

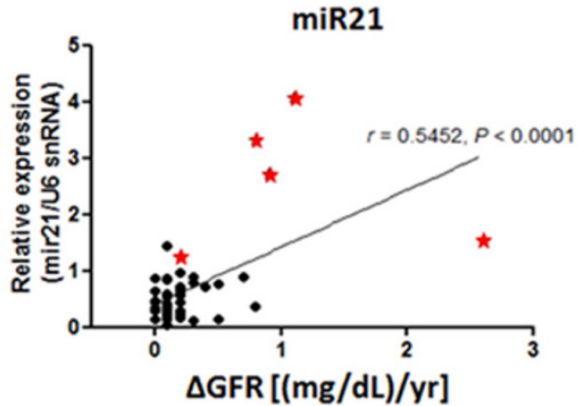

C

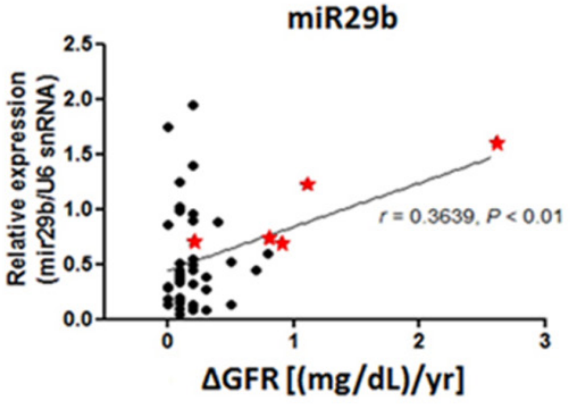

E

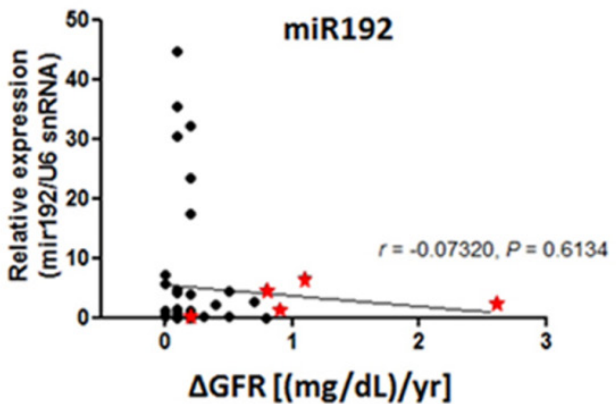

B

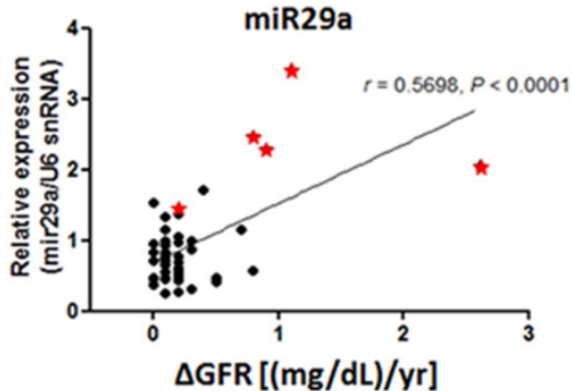

D

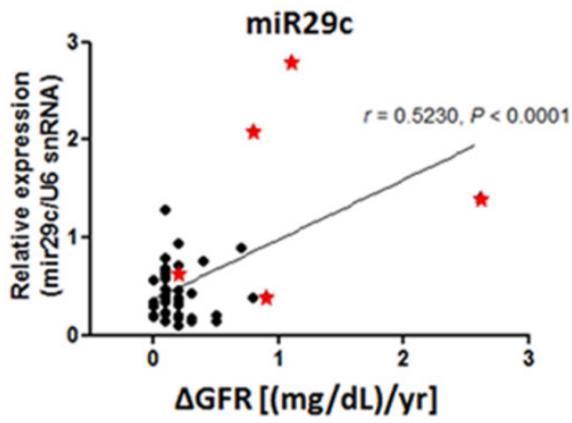

Figure 3. Correlation of serum miR levels with disease progression in patients with diabetic nephropathy. Pearson's correlation coefficient analysis was performed and $P<0.05$ is considered as significant difference. The result showed that the subjects with greater level of serum miR-21 and miR-29 family exhibited more rapid creatinine changes in contrast to those with lower miR levels. The $\mathrm{X}$-axis represented changing rates of creatinine over 1 -year follow-up and the $\mathrm{Y}$-axis referred to the relative miR level (versus U6 snRNA). Asterisks represent subjects with greater candidate miR level.

\section{Discussion}

DN accounts for a significantly increased morbidity and mortality in T2DM patients. Although microalbuminuria is regarded as the gold standard for diagnosis of an early and reversible DN, the sensitivity to precisely detect disease progression remains unsatisfied [19]. Therefore, exploring more sensitive diagnostic markers for better monitoring of DN pathogenesis could largely facilitate risk stratification of the disease and enable the earlier diagnosis and more efficient intervention. In the present study, the potential to utilize circulating miRs as prognostic indicators in T2DM patients with differential DN stages to define or to predict $\mathrm{DN}$ progression was determined. Using quantitative RT-PCR analysis for DN related miRs, the results demonstrated that the level of miR-21 and miR-29a was significantly elevated in subjects with overt proteinuria compared with the patients diagnosed with either normal albuminuria or microalbuminuria.
Interestingly, among all candidate miRs, miR-192 level showed good sensitivity to diagnose established DN progression, namely between subjects with microalbuminuria and overt proteinuria. In contrast, by using the eGFR as a classification indicator, it was found that only miR-21 level was significantly up-regulated among the groups indicating that the miR-21 possibly serves as a more sensitive circulating miR to reflect early renal dysfunction.

Recent study defined the importance of miRs in normal renal development and function and various renal diseases suggesting specific renal miRs might be good targets to monitor DN pathogenesis [20]. For example, miR-192 is specifically expressed in kidney and its level is up-regulated in streptozotocin (STZ)-induced DM and in $\mathrm{db} / \mathrm{db}$ mice. Further analysis found that miR-192 suppressed the translation of SIP1/ E-box repressors ZEB2, a transcriptional repressor that binds to the E-box in the Collagen1a2 (Col1a2) gene, leading to elevated collagen deposition in vivo indicating a role of 
miR-192 in the development of the matrix accumulation observed in DN [21]. In addition, Krupa et al showed miR-192 was significantly lower in patients with advanced DN, correlating with tubulointerstitial fibrosis and low GFR concluding that a decrease in miR-192 is associated with increased renal fibrosis in vivo [22]. Other DN associated miRs such as miR-21 and miR-29 were also described. The level of miR-21 was down-regulated in early DN while overexpression of miR-21 inhibits glomerular mesangial cell proliferation under conditions of elevated glucose. Ectopic miR-21 expression in vivo resulted in decreased albuminuria in diabetic $\mathrm{db} / \mathrm{db}$ mice suggesting its role in maintaining renal functions in response to high glucose condition [23]. In molecular basis, miR-21 prevented mesangial hypertrophy by targeting the PTEN/PI3K/Akt pathway in vivo and in vitro and overexpression of miR-21 enhanced renal fibrosis in vitro in response to Transforming growth factor- $\beta 1$ (TGF- $\beta 1$ ) treatment implying the importance of miR-21 in regulating renal fibrosis and cell apoptosis [24]. Furthermore, recent reports have also found that miR-29 was downregulated in the fibrotic kidney of obstructive nephropathy and was negatively regulated by TGF- $\beta 1$ via Smad3 signaling pathway as Smad3 physically bound the miR-29 promoter and repressed miR-29 expression, thereby promoting collagen matrix expression [25]. Knock-down of miR-29c significantly reduced albuminuria and kidney mesangial matrix accumulation in the $\mathrm{db} / \mathrm{db}$ mice model in vivo, and prevented high glucose-induced cell apoptosis whereas forced expression of miR-29c strongly induced podocyte apoptosis [26]. In a with our findings, a recent study showed that urinary miR-29a, but not miR-29b and miR-29c, was elevated in T2DM subjects with microalbuminuria and overt proteinuria compared to those with normoalbuminuria [27]. On the contrary, Lv et al demonstrated that urinary levels of miR-29 and miR-200 family were decreased in patients with the chronic kidney diseases (CKD), including biopsy-defined DN, whereas the miR-29c expression was positively correlated with eGFR and negatively associated with degree of tubulointerstitial fibrosis [28]. The inconsistent findings might result from the diverse etiology of CKD populations included in the cohort while the small number of the DN samples $(\mathrm{N}=3)$ also limited further interpretation of the study. In addition, it has been found that serum miR levels do not always consistent with the levels of urinary miRs highlighting the importance of sample selection to explore useful DN biomarkers.

In cellular basis, miRs are indispensable in regulating normal development and in maintaining organ function and homeostasis [29]. While the aberrant miR level is observed in mice with kidney fibrosis; the ablation of Dicer protein, a key miR biogenetic mediator, in podocytes led to proteinuria and glomerulosclerosis [30]. Previous studies indicated that decreased miR-29 level could result in DN and IgA nephropathy [31]. Nevertheless, an inconsistent result showed that the increased miR-29c level was detected in the kidney glomeruli from $\mathrm{db} / \mathrm{db}$ mice and deficient miR-29c level could ameliorate the DN progression via the reduction of cell apoptosis and decreased ECM expression under diabetic condition in vivo [26]. Interestingly, the TGF- $\beta$ mediated level of miR-21, miR-29 and miR-192 played differential roles in modulating renal fibrosis; miR-21 and miR-192 amplified the TGF- $\beta$ signaling and promoted fibrosis as miR-29 reduced fibrosis by inhibition of the deposition of ECM [32]. The potential regulatory role of miR-192 mediated circuits during DN pathogenesis was further emphasized, showing that miR-192 could amplify TGF- $\beta 1$ signaling thereby accelerating DN [33]. Under the treatment of anti-miR-192 in STZ-induced diabetic mice, the significantly decreased TGF- $\beta$ expression, collagen and fibronectin accumulation as well as the attenuated proteinuria were detected in diabetic kidneys implying the potential of miR-192 blockage for DN treatment [34]. Several lines of evidence have revealed the potential miR-mediated downstream molecular players for DN pathogenesis. For example, miR-21 targeted down-regulation of tissue inhibitors of metalloproteinase 3 (TIMP3) as well as the miR-21 regulated PPARa-mediated lipid metabolic pathway both play important roles for renal fibrosis [35, 36]. A recent study also found that in vivo administration of miR-21 knockdown plasmid into the diabetic kidneys of $\mathrm{db} / \mathrm{db}$ mice could rescue the microalbuminuria, renal fibrosis and inflammation via the restoration of a TGF- $\beta 1$ repressor mothers against decapentaplegic homolog 7 (SMAD7), revealing a miR-21 targeted therapeutic strategy for DN treatment [37].

There were several clinical indicators to monitor DN progression. For instance, early studies indicated that the presence of microalbuminuria in $80 \%$ of T1DM patients could be a predictive of disease progression [38]; nevertheless, the other analysis showed that only around $30 \%$ of microalbuminuric patients progressed to overt DN after 10-year follow-up [39]. Moreover, no definite correlation for the low-eGFR subjects with a long DM history to progression from normoalbuminuria to microalbuminuria was established. In addition, more recent evidence suggested that advanced histological alterations in the glomerular basement membrane may already exist when microalbuminuria is first detected, suggesting microalbuminuria might not be 
sensitive enough to determine $\mathrm{DN}$ onset/progression [40]. The utilization of microalbuminuria for detection of $\mathrm{DN}$ becomes even more complicated when a very recent study demonstrated that more than half of T2DM patients with overt proteinuria obtained remission to microalbuminuria after the intensive DM treatment in a primary care setting [41]. The detection of tubulointerstitial fibrosis by renal biopsy was also widely accepted as one of the histopathological hallmarks of progressive $\mathrm{DN}$ and the degree of tubulointerstitial fibrosis could even be used to predict renal function [42]. However, renal biopsy is invasive with potential complications making repeated monitoring become practically difficult. Hence, reliable noninvasive biomarkers reflecting disease severity are urgently needed in the clinical management of patients with CKD. Our data showed that $\mathrm{DN}$ progressors, defined as patients with a more rapid change of eGFR or ACR, exhibited significantly greater serological levels of miR-21 and miR-29 family, but not miR-192, in comparison with non-progressors, revealing the significance to use serum miRs to identify DN advancement. Pezzolesi et al. showed that and miR-21 were significantly associated with the increased risk of ESRD while miR-29a were significantly associated with protection against rapid progression in T1DM patients [43]. In agreement with our finding, a recent study identified a number of uninary miRs that have not previously been associated with renal pathology in T1DM patients with different stages of albuminuria/DN. Among those DN-related miRs, miR-29 is differentially expressed between patients who developed overt DN after decades relative to patients who did not [43], although a null differential significance of miR level between DN progressors and non-progressors was found in another study [22].

The current study revealed for the first time, to best of our knowledge, that miR-21, miR-29a/b/c and miR-192 could possibly reflect $\mathrm{DN}$ pathogenesis; however, there were several limitations in this study. First, the sample size is relatively small. Second, renal biopsy had only been performed in a few subjects, making it difficult to elucidate the correlation between miR level and renal histopathological condition, such as degree of renal fibrosis. Lastly, the lack of repeated measurements for candidate miRs during the follow-up period restricted the precise interpretations for the importance of tested miRs during DN progression. In summary, we reported that serological miR-21, miR-29a and miR-192 are significantly elevated in T2DM patients with aggressive DN, based either on ACR or eGFR classification. The progressors showed significantly greater levels of miR-21 and miR-29 family in comparison with non-progressors.
Our result implied that these miRs may serve as early indicators of DM-mediated renal pathology, which can be of importance in the aspect of preventive medicine. A larger cohort would be required to warrant our findings and to clarify the potential utility of these miRs in early diagnosis, risk stratification of progression and treatment outcomes.

\section{Supplementary Material}

Supplementary tables and figures. http://www.medsci.org/v13p0457s1.pdf

\section{Acknowledgments}

This work is supported by a grant from the Taipei City Hospital/Department of Health, Taipei City Government (100TPECH06 to W-C Li) as well as a grant from Ministry of Science and Technology, Taiwan (Most-103-2314-B-010-024-MY3 to W-C Li). The authors would also like to thank Mrs Courtney Anne Curtis for critical review and English corrections for the manuscript.

\section{Competing interests}

The authors declare no competing interest exists.

\section{References}

1. Yang WC, Hwang SJ, Chiang SS, Chen HF, Tsai ST. The impact of diabetes on economic costs in dialysis patients: experiences in Taiwan. Diabetes research and clinical practice. 2001;54 (Suppl 1):S47-54.

2. Boyle JP, Thompson TJ, Gregg EW, Barker LE, Williamson DF. Projection of the year 2050 burden of diabetes in the US adult population: dynamic modeling of incidence, mortality, and prediabetes prevalence. Population health metrics. 2010;8:29.

3. Bell DS. Diabetic cardiomyopathy. A unique entity or a complication of coronary artery disease? Diabetes care. 1995;18:708-714.

4. Ahlqvist E, van Zuydam NR, Groop LC, McCarthy MI. The genetics of diabetic complications. Nature Reviews Nephrology. 2015; 11:277-287.

5. Reidy K, Kang HM, Hostetter T, Susztak K. Molecular mechanisms of diabetic kidney disease. The Journal of clinical investigation. 2014;124:2333-2340.

6. Oberg BP, McMenamin E, Lucas FL et al. Increased prevalence of oxidant stress and inflammation in patients with moderate to severe chronic kidney disease. Kidney international. 2004;65:1009-1016.

7. Jerums G, Premaratne E, Panagiotopoulos S, Clarke S, Power DA, MacIsaac RJ. New and old markers of progression of diabetic nephropathy. Diabetes research and clinical practice. 2008;82 (Suppl 1):S30-37.

8. Jerums G, Premaratne E, Panagiotopoulos S, MacIsaac RJ. The clinical significance of hyperfiltration in diabetes. Diabetologia. 2010;53:2093-2104.

9. Levey AS, Becker C, Inker LA. Glomerular filtration rate and albuminuria for detection and staging of acute and chronic kidney disease in adults: a systematic review. JAMA : the journal of the American Medical Association. 2015;313:837-846.

10. Alvarez ML, Khosroheidari M, Kanchi Ravi R, DiStefano JK. Comparison of protein, microRNA, and mRNA yields using different methods of urinary exosome isolation for the discovery of kidney disease biomarkers. Kidney international. 2012;82:1024-1032.

11. Conserva F, Pontrelli P, Accetturo M, Gesualdo L. The pathogenesis of diabetic nephropathy: focus on microRNAs and proteomics. Journal of nephrology. 2013;26:811-820.

12. Mendell JT. MicroRNAs: critical regulators of development, cellular physiology and malignancy. Cell cycle. 2005;4:1179-1184.

13. Gilad S, Meiri E, Yogev Y, et al. Serum microRNAs are promising novel biomarkers. PloS one. 2008;3:e3148.

14. Miranda KC, Huynh T, Tay $\mathrm{Y}$, et al. A pattern-based method for the identification of MicroRNA binding sites and their corresponding heteroduplexes. Cell. 2006;126:1203-1217.

15. Baek D, Villen J, Shin C, Camargo FD, Gygi SP, Bartel DP. The impact of microRNAs on protein output. Nature. 2008;455:64-71.

16. Bhattacharyya SN, Habermacher R, Martine U, Closs EI, Filipowicz W. Relief of microRNA-mediated translational repression in human cells subjected to stress. Cell. 2006;125:1111-1124. 
17. Guo H, Ingolia NT, Weissman JS, Bartel DP. Mammalian microRNAs predominantly act to decrease target mRNA levels. Nature. 2010;466:835-840.

18. Xu J, Zhao J, Evan G, Xiao C, Cheng Y, Xiao J. Circulating microRNAs: novel biomarkers for cardiovascular diseases. Journal of molecular medicine. 2012;90:865-875.

19. Trionfini P, Benigni A, Remuzzi G. MicroRNAs in kidney physiology and disease. Nature Reviews Nephrology. 2015;11:23-33.

20. Li JY, Yong TY, Michael MZ, Gleadle JM. Review: The role of microRNAs in kidney disease. Nephrology. 2010;15:599-608.

21. Kato M, Zhang J, Wang M, et al. MicroRNA-192 in diabetic kidney glomeruli and its function in TGF-beta-induced collagen expression via inhibition of E-box repressors. Proceedings of the National Academy of Sciences of the United States of America. 2007;104:3432-3437.

22. Krupa A, Jenkins R, Luo DD, Lewis A, Phillips A, Fraser D. Loss of MicroRNA-192 promotes fibrogenesis in diabetic nephropathy. Journal of the American Society of Nephrology : JASN. 2010;21:438-447.

23. Zhang $\mathrm{Z}$, Peng $\mathrm{H}$, Chen $\mathrm{J}$, et al. MicroRNA-21 protects from mesangial cell proliferation induced by diabetic nephropathy in $\mathrm{db} / \mathrm{db}$ mice. FEBS letters. 2009;583:2009-2014.

24. Kantharidis P, Wang B, Carew RM, Lan HY. Diabetes complications: the microRNA perspective. Diabetes. 2011;60:1832-1837.

25. Qin W, Chung AC, Huang XR, et al. TGF-beta/Smad 3 signaling promotes renal fibrosis by inhibiting miR-29. Journal of the American Society of Nephrology : JASN. 2011;22:1462-1474.

26. Long J, Wang Y, Wang W, Chang BH, Danesh FR. MicroRNA-29c is a signature microRNA under high glucose conditions that targets Sprouty homolog 1, and its in vivo knockdown prevents progression of diabetic nephropathy. The Journal of biological chemistry. 2011;286:11837-11848.

27. Peng $\mathrm{H}$, Zhong $\mathrm{M}$, Zhao $\mathrm{W}$, et al. Urinary miR-29 correlates with albuminuria and carotid intima-media thickness in type 2 diabetes patients. PloS one. 2013;8:e82607.

28. Lv LL, Cao YH, Ni HF, et al. MicroRNA-29c in urinary exosome/microvesicle as a biomarker of renal fibrosis. American journal of physiology Renal physiology. 2013;305:F1220-1227.

29. Wessely O, Agrawal R, Tran U. MicroRNAs in kidney development: lessons from the frog. RNA biology. 2010;7:296-299.

30. Shi S, Yu L, Chiu C, et al. Podocyte-selective deletion of dicer induces proteinuria and glomerulosclerosis. Journal of the American Society of Nephrology : JASN. 2008;19:2159-2169.

31. Wang G, Kwan BC, Lai FM, Chow KM, Li PK, Szeto CC. Urinary miR-21, miR-29, and miR-93: novel biomarkers of fibrosis. American journal of nephrology. 2012;36:412-418.

32. Chung AC, Lan HY. MicroRNAs in renal fibrosis. Frontiers in physiology. 2015;6:50.

33. Kato M, Arce L, Wang M, Putta S, Lanting L, Natarajan R. A microRNA circuit mediates transforming growth factor-beta1 autoregulation in renal glomerular mesangial cells. Kidney international. 2011;80:358-368.

34. Putta $S$, Lanting $L$, Sun $G$, Lawson $G$, Kato $M$, Natarajan $R$. Inhibiting microRNA-192 ameliorates renal fibrosis in diabetic nephropathy. Journal of the American Society of Nephrology : JASN. 2012;23:458-469.

35. Chau BN, Xin C, Hartner J, et al. MicroRNA-21 promotes fibrosis of the kidney by silencing metabolic pathways. Science translational medicine. 2012;4:121ra18.

36. Fiorentino L, Cavalera M, Mavilio M, et al. Regulation of TIMP3 in diabetic nephropathy: a role for microRNAs. Acta diabetologica. 2013:50:965-969.

37. Zhong X, Chung AC, Chen HY, et al. miR-21 is a key therapeutic target for renal injury in a mouse model of type 2 diabetes. Diabetologia. 2013;56:663-674.

38. Rossing P, Hougaard P, Parving HH. Progression of microalbuminuria in type 1 diabetes: ten-year prospective observational study. Kidney international. 2005;68:1446-1450.

39. Allen KV, Walker JD. Microalbuminuria and mortality in long-duration type 1 diabetes. Diabetes care. 2003;26:2389-2391.

40. Dronavalli S, Duka I, Bakris GL. The pathogenesis of diabetic nephropathy. Nature clinical practice Endocrinology \& metabolism. 2008;4:444-452.

41. Yokoyama H, Araki S, Honjo J, et al. Association between remission of macroalbuminuria and preservation of renal function in patients with type 2 diabetes with overt proteinuria. Diabetes care. 2013;36:3227-3233.

42. Mise $\mathrm{K}$, Hoshino J, Ueno $\mathrm{T}$, et al. Impact of tubulointerstitial lesions on anaemia in patients with biopsy-proven diabetic nephropathy. Diabetic medicine : a journal of the British Diabetic Association. 2015;32:546-555.

43. Argyropoulos C, Wang K, McClarty S, et al. Urinary microRNA profiling in the nephropathy of type 1 diabetes. PloS one. 2013;8:e54662. 